\title{
]jfis
}

\section{Performance Analysis of Denoising Algorithms for Human Brain Image}

Nishant Chauhan and Byung-Jae Choi

Department of Electronic Engineering, Daegu University, Gyeongsan, Korea

\begin{abstract}
Digital images have an important role in areas like X-ray, face recognition, and so on. Different image capturing devices inherit different types of noises. Sometimes low image quality is an obstacle for analysis and measurement. The denoising of an image is an important task. An ideal denoising technique should be able to remove the noise while preserving the quality of the image. In this paper, we examine four noise removal algorithms and present a performance analysis of PSNR and RMSE of several filters for various noises and simulate the performance using MATLAB for human brain image.
\end{abstract}

Keywords: Human brain image, Denoising, Filter, PSNR, RMSE, Performance analysis

\section{Introduction}

The noise is a fundamental attribute which is present in all images. Hence digital images and their analysis for making them noise free are having a significant role in fields such as traffic detection, face detection, remote sensing, astronomy, reading medical images, etc. Excessive noise reduces the visibility of some structure and object; especially it is more severely affected in case of medical images (magnetic resonance imaging [MRI] and X-ray). Therefore, the denoising of medical images is important for not only just to eliminate the noise but also to limit it to a clinically acceptable level. Optimization of denoising procedures requires the study and performance analysis of denoising algorithms with respect to the noise present in the image. Hence while selecting an appropriate denoising algorithm; one should also focus in maintaining their edges and originality.

Digital images have an important role in areas like television, ultrasound, X-ray, face recognition, and so on. Different image capturing devices consist different types of noises [1]. Noise is generally defined as the values of random pixels in recorded image which are different from the true intensities of real image [2]. Sometimes low image quality is an obstacle for analysis and measurement. So the denoising of an image is an important task. An ideal denoising techniques should be able to remove the noise while preserving the quality of the image [2, 3]. In case of medical fields, the role of medical images has an important role in pathological diagnosis or surgical intervention. However, MRI and ultrasound (US) images normally affected by noise which was produced by electrical fluctuation or radiation which make it difficult for pathological diagnosis.

Denoising and enhancement of the medical images can be useful in feature extraction, image restoration and reducing distortion of complex images like MRI of human brain [4-7]. The noise infected may be Gaussian noise, speckle noise, Poisson noise, etc. Sometime the 
Table 1. Various types of noises, and their sources and effects

\begin{tabular}{|c|c|c|c|c|}
\hline \multirow{2}{*}{ Parameters } & \multicolumn{4}{|c|}{ Types of noises } \\
\hline & Gaussian & $\begin{array}{l}\text { Salt \& } \\
\text { Pepper }\end{array}$ & Poisson & Speckle \\
\hline Source & $\begin{array}{c}\text { Occurs } \\
\text { during } \\
\text { image } \\
\text { acquisition } \\
\text { /electronic } \\
\text { noise }\end{array}$ & $\begin{array}{c}\text { Sudden } \\
\text { disturbances } \\
\text { in the image } \\
\text { signal/data } \\
\text { drop noise }\end{array}$ & $\begin{array}{l}\text { Discrete } \\
\text { nature of } \\
\text { electric } \\
\text { charge }\end{array}$ & $\begin{array}{c}\text { Modeled } \\
\text { by random } \\
\text { values } \\
\text { multiplied } \\
\text { by pixel } \\
\text { values }\end{array}$ \\
\hline Effect & Blur edges & $\begin{array}{c}\text { Black and } \\
\text { white dots in } \\
\text { whole image }\end{array}$ & $\begin{array}{c}\text { Discrete } \\
\text { dots/lines } \\
\text { of white } \\
\text { or black } \\
\text { color }\end{array}$ & $\begin{array}{c}\text { Similar as } \\
\text { Gaussian } \\
\text { noise in } \\
\text { radar, } \\
\text { laser, } \\
\text { acoustics } \\
\text { images }\end{array}$ \\
\hline
\end{tabular}

capturing device itself have some salt and pepper noise which affects the medical image badly. To remove the noise or distortion it very important to select the appropriate filtering technique. The main objective of this paper to compare the performance of various known noise filters (average filter, median filter, Gaussian filter, and Wiener filter) in case of medical imaging under MATLAB environment [8]. A test image of human brain MRI has been chosen for experiment [9]. Two measurement parameter root mean square error (RMSE) and peak signal to noise ratio (PSNR) were used to compare the results of four noise filters.

\section{Several Noises and Filters}

\subsection{Several Noises}

Noises are unwanted and undesirable effects on images. It reduces the true identity of image and shows effects like blurred corners, unseen lines, unknown dots, etc. To apply the denoising techniques it is very important to understand the noise model and their characteristics.

Table 1 shows several types of noises and their sources and effects [10, 11].

The detailed noise models and their characteristics are described as follows.

\subsubsection{Gaussian noise}

Gaussian noise is also called amplifier noise which is independent of the signal intensity. It is distributed evenly over the whole image because its probability distribution density func- tion (PDF) is equal to the normal distribution [1]. It can be expressed by Eq. (1)

$$
p(z)=\frac{1}{\sqrt{2 \pi \sigma}} e^{\frac{-(z-\mu)^{2}}{2 \sigma^{2}}} .
$$

\subsubsection{Salt and Pepper noise}

Salt and Pepper noise is also referred as impulse noise or data drop noise because some pixels in the image dropped their original values. In 8-bit transmission system, some pixels become white as salt (maximum gray level 255) and some become black as pepper (minimum gray level 0 ).

We can see those pixels in image as black and white dots. The source of Salt and Pepper noise is disturbance in image signal, over heated/faulty components or dust particles in image acquisition source [1, 3, 12]. Its PDF is given by Eq. (2):

$$
P(z)= \begin{cases}P_{a} & \text { for } z=a \\ P_{b} & \text { for } z=b \\ 0 & \text { otherwise }\end{cases}
$$

\subsubsection{Poisson noise}

Poisson noise is also called shot noise. It occurs when the number of photos sensed by a sensor is not calculative or insufficient to produce the true identity of source data [12]. The $\mathrm{X}$-ray and gamma ray sources emit many photos per unit time and have random fluctuations. In the medical images, this noise is the result of gathered spatial and temporal randomness. The random pixels are suffered by independent noise values. This noise has root mean square value proportional to square root of the intensity of the image [10, 12]. The PDF for Poisson noise is given as Eq. (3):

$$
p(z)=\frac{e^{-\boldsymbol{\lambda}} \boldsymbol{\lambda}^{x}}{z !} \text { for } \lambda>0 \text { and } x=0,1,2 \ldots
$$

\subsubsection{Speckle noise}

Speckle noise mostly occurs in coherent imaging systems (CIS) and synthetic aperture radars (SAR). The true pixel values are multiplied by random values which make it multiplicative in nature which results increasing the mean gray level of image. The distribution of Speckle noise can be represented by Eq. (4):

$$
J=I+n \times I,
$$

where $I$ denote the original image, $J$ is the captured image and $n$ is the uniformly distributed random noise with zero mean. 
Table 2. Functions used in the denoising algorithms

\begin{tabular}{|c|c|}
\hline Filter & Function \\
\hline \multirow{2}{*}{ Average } & $\sum_{s=-a}^{a} \sum_{t=-b}^{b} \omega(s, t) \cdot f(x+s, y+t)$ \\
\hline & $g(x, y)=\overline{\sum_{s=-a}^{a} \sum_{t=-b}^{b} \omega(s, t)}$ \\
\hline Median & $f(x, y)=$ Median $\{g(s, t)\}$, where $(s, t) \in s_{x y}$ \\
\hline Gaussian & $\begin{array}{l}\omega_{i j}=\frac{1}{2 \pi \sigma^{2}} e^{\left\{\frac{-\left(i^{2}+j^{2}\right)}{2 \sigma^{2}}\right\}} \\
\text { where } \sigma \text { is standard deviation }\end{array}$ \\
\hline Wiener & $\begin{array}{l}G(u, v)=\frac{H^{*}(u, v)}{|H(u, v)|^{2} \cdot P_{s}(u, v)+P_{n}(u, v)}, \\
\text { where } \\
\mathrm{H}^{*}(\mathrm{u}, \mathrm{v})=\begin{array}{l}\text { complex conjugate of } \\
\text { degradation function, }\end{array} \\
\mathrm{P}_{\mathrm{n}}(\mathrm{u}, \mathrm{v})=\begin{array}{l}\text { power spectral density of } \\
\text { noise, }\end{array} \\
\mathrm{P}_{\mathrm{s}}(\mathrm{u}, \mathrm{v})=\begin{array}{l}\text { power spectral density of } \\
\text { non-degraded image, }\end{array} \\
\mathrm{H}(\mathrm{u}, \mathrm{v})=\begin{array}{l}\text { degradation function } \\
\text { degration }\end{array}\end{array}$ \\
\hline
\end{tabular}

\subsection{Several Noise Filters}

Study of noise model is very important in digital image processing as noise has been seen at different stages like image acquisition and transmission. Similarly, a denoising of the image is also very important step in image processing.

So, the prior knowledge of noise models might be to the user to choose the appropriate technique of denoising.

Denoising algorithms can be linear as well as non-linear, where the difference lies between the fast processing and preserving the details of subject images [10]. Linear algorithms are sufficiently fast whereas non-linear are capable of image preservation. Some image denoising filters are shown in Table 2.

\subsubsection{Average filter}

The average filter is like a smoothing filter. It replaces the value of every pixel in an image with the average of the gray levels defined by the filter mask. Its filter mask computes the average of neighboring pixels and replaces the value with centered pixel value. The undesirable effects of the average filter are blurred image. Average filtering example using $3 \times 3$ filter mask can be understood by Figure 1 .

\subsubsection{Median filter}

In median filtering, it replaces the value of a pixel by the median of the gray levels in the neighborhood of the pixel. The main

Input Average $=(1+4+0+1+4+0+2+2+4) / 9=2$
\begin{tabular}{|r|c|c|c|c|c|c|r|r|r|r|r|}
\hline 1 & 4 & 0 & 1 & 3 & 1 \\
\hline 1 & 4 & 0 & 1 & 3 & 1 & 1 \\
\hline 2 & 2 & 4 & 2 & 2 & 3 & 3 \\
\hline 1 & 1 & 0 & 1 & 0 & 1 & 0 & 0 \\
1 & 1 & 2 & 1 & 0 & 2 & 2 & 2 \\
\hline 1 & 1 & 2 & 6 & 4 & 2 & 4 & 4 \\
\hline 1 & 1 & 3 & 2 & 1 & 4 & 0 & 0 \\
\hline
\end{tabular}

Figure 1. Example of average filtering using $3 \times 3$ filter mask.

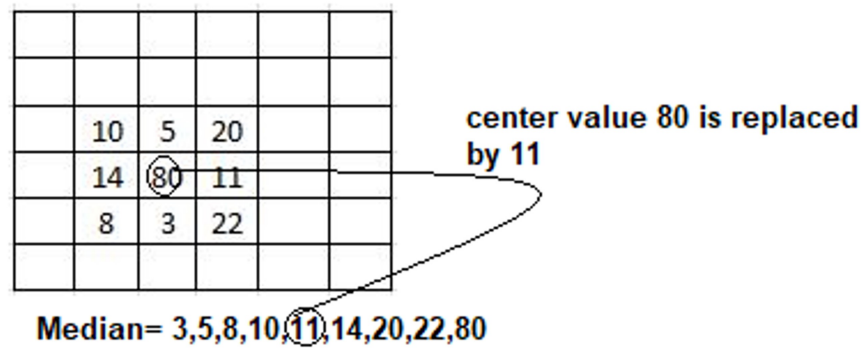

Figure 2. Example of median filtering using $3 \times 3$ filter mask.

$\left\{\begin{array}{l}\frac{1}{2 \pi 5.5^{2}} e^{\left\{\frac{-\left(1^{2}+1^{2}\right)}{2 \times 5.5^{2}}\right\}} \frac{1}{2 \pi 5.5^{2}} e^{\left\{\frac{-\left(0^{2}+1^{2}\right)}{2 \times 5.5^{2}}\right\}} \frac{1}{2 \pi 5.5^{2}} e^{\left\{\frac{-\left(1^{2}+1^{2}\right)}{2 \times 5.5^{2}}\right\}} \\ \frac{1}{2 \pi 5.5^{2}} e^{\left\{\frac{-\left(1^{2}+0^{2}\right)}{2 \times 5.5^{2}}\right\}} \frac{1}{2 \pi 5.5^{2}} e^{\left\{\frac{-\left(0^{2}+0^{2}\right)}{2 \times 5.55^{2}}\right\}} \frac{1}{2 \pi 5.5^{2}} e^{\left\{\frac{-\left(1^{2}+0^{2}\right)}{2 \times 5.5^{2}}\right\}} \\ \frac{1}{2 \pi 5.5^{2}} e^{\left\{\frac{-\left(1^{2}+1^{2}\right)}{2 \times 5.5^{2}}\right\}} \frac{1}{2 \pi 5.5^{2}} e^{\left\{\frac{-\left(0^{2}+1^{2}\right)}{2 \times 5.5^{2}}\right\}} \frac{1}{2 \pi 5.5^{2}} e^{\left\{\frac{-\left(1^{2}+1^{2}\right)}{2 \times 5.5^{2}}\right\}}\end{array}\right\}$

Figure 3. Example of Gaussian filtering using $3 \times 3$ filter mask.

advantage of this filter is it preserve sharp edges and avoids blurring the image. A median filtering example using $3 \times 3$ filter mask presented in Figure 2.

\subsubsection{Gaussian filter}

The Gaussian filter removes the high-frequency components from the image (low-pass filter). It is a smoothing filter in the $2 \mathrm{D}$ convolution operation that is used to remove noise and blur image. The degree of smoothing is controlled by standard variance. For example in Figure 3, for standard variance 5.5 and $3 \times 3$ Gaussian kernel, the pixel values can be calculated as:

\subsubsection{Wiener filter}

This is a good deblurring linear filter. It provides a solution for stationary signals and signal estimation problems. It also esti- 
mates the desired signal using statistical approach and reduces the amount of noise present in a signal by comparison with an estimation of the desired noiseless signal. This filter performs well for noises like Poisson noise and Speckle noise [11].

\section{Methods}

Comparing restoration results requires a measure of image quality. The commonly used measures are MSE and PSNR [13]. The MSE between two images $I$ and $K$ is defined by Eq (5):

$$
M S E=\frac{1}{m n} \sum_{i=0}^{m-1} \sum_{j=0}^{n-1}[I(i, j)-K(i, j)]^{2},
$$

where $I$ and $K$ are original and denoised images of size $m$ and $n$, respectively.

The PSNR in $\mathrm{dB}$ is defined as (Eq. (6)):

$$
P S N R=10 \cdot \log _{10}\left(\frac{M A X_{i}^{2}}{M S E}\right),
$$

where $\mathrm{MAX}_{\mathrm{i}}{ }^{2}$ is the maximum possible pixel value of the image ( 8 bits) and $I$ and $K$ are the original and denoised images, respectively.

RMSE has the same units as MSE and its quantity has been estimated as the square root of MSE [13]. It gives a relatively high weight to large errors. It can be calculated by square root of MSE (Eq. (5)). It can be expressed by Eq. (7).

$$
R M S E=\sqrt{M S E} .
$$

\section{Dynamics}

The performance analysis is done by the following steps (Figure 4).

A sample image for the performance analysis is a human brain MRI image as shown in Figure 5. All four types of noises are first induced to human brain image.

The reference image is converted to a gray scale image. Four types of noises, Gaussian noise, Salt \& Pepper noise, Poisson noise and Speckle noise are added using MATLAB (Figures 6-9).

The Gaussian noise is distributed in whole image, however the Salt \& Paper noise is independent and uncorrelated to image pixels. So, it is can be seen by black and white particles. The reason behind the occurrence of this noise is sudden changes of image signals. Poisson noise is also termed as shot noise, and it

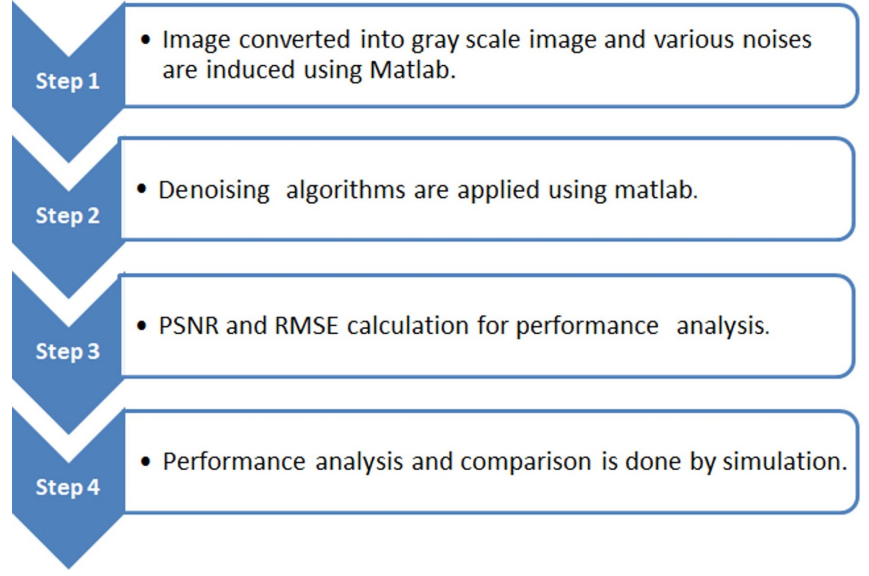

Figure 4. Performance analysis process.

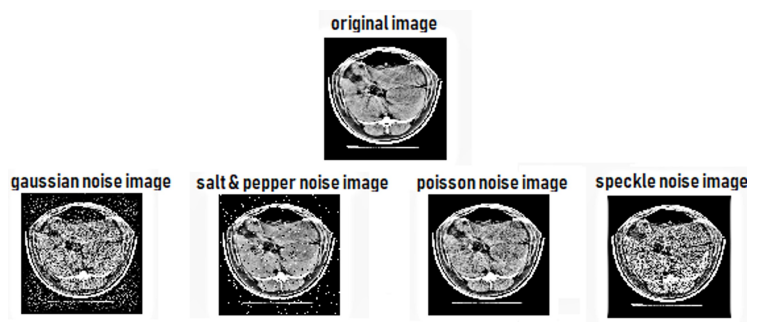

Figure 5. Original image and several noised images.

follows Poisson.

In this noise, the different pixels were affected by independent noise intensity, however in Speckle noise the gray level of the image was affected.

\section{Performance Analysis by Four Filters}

Denoising was performed using four filters: average filter, median filter, Gaussian filter, and Wiener filter.

The performance of average filter is satisfactory in these results as it takes the average value of neighborhood pixels in mask and replaces it with current value. It has some blur effect on image. It is mostly using to remove the irrelevant details from an image (Figure 6).

The quality of image filtered by median filter is better than other filtered images. The functionality of median filter is based on ranking of pixel values in filter region and its center value is replaced the median value (Figure 7).

Gaussian filter removes Gaussian noise, but it is not good for images having Poisson noise. The reason behind this is Gaussian distribution is continuous whereas the Poisson distribution is discrete. Median and Wiener filter have better performance (Figure 8). 


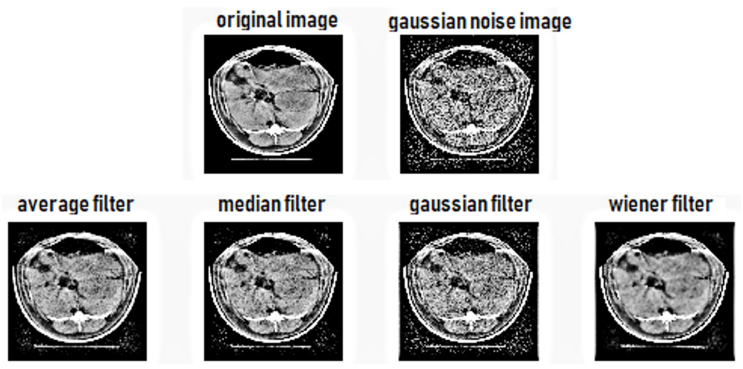

Figure 6. Performance of four filters on Gaussian noise image.

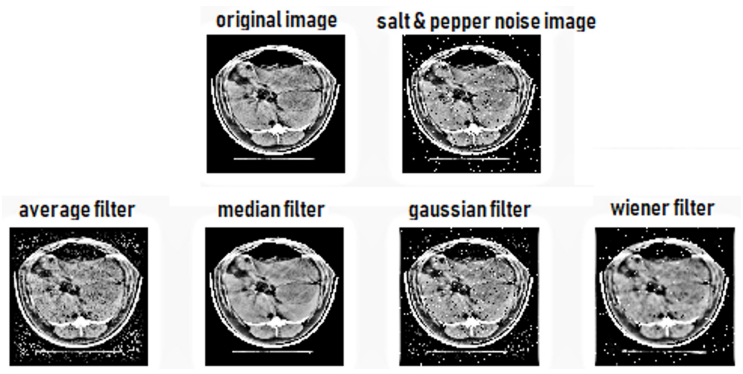

Figure 7. Performance of four filters on Salt and Pepper noise image.

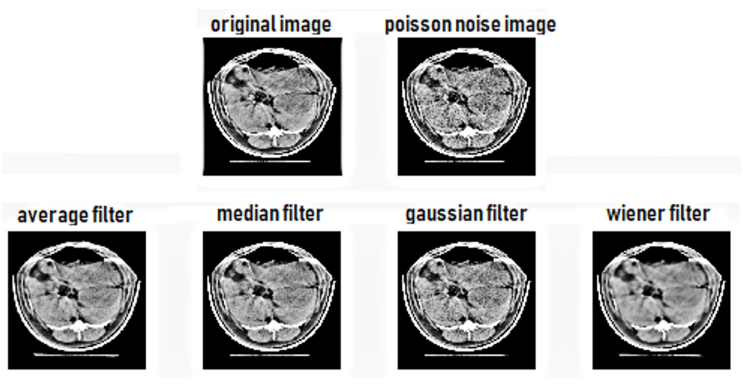

Figure 8. Performance of four filters on Poisson noise image.
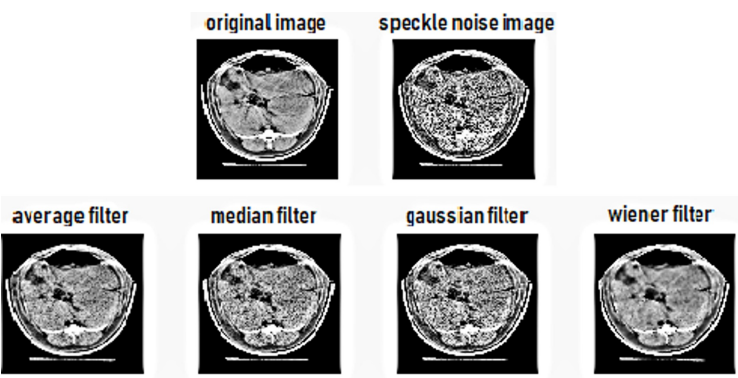

Figure 9. Performance of four filters on Speckle noise image.

For Poisson noise and Speckle noise, Wiener filter eliminates a good amount of noise because it minimizes the MSE between the estimated random processes (Figure 9). The benefit of minimizing the MSE randomly Wiener filter reduces the relative insignificance in many cases of electrical conduction (Poisson noise).
Table 3. Results of PSNR (in dB)

\begin{tabular}{ccccc}
\hline \multirow{2}{*}{ Noises } & \multicolumn{4}{c}{ Filters } \\
\cline { 2 - 5 } & Average & Median & Gaussian & Wiener \\
\hline Gaussian & 31.7115 & 30.4332 & 34.5571 & 31.2017 \\
Salt \& & 28.0278 & 34.64 & 24.7715 & 23.5598 \\
Pepper & & & 30.9068 & 31.1984 \\
\hline Poisson & 25.5247 & 29.5879 & 22.7615 & 29.218 \\
\hline Speckle & 27.613 & 25.7222 & 25 \\
\hline
\end{tabular}

Speckle noise is caused by coherent processing of backscattered signals from multiple distributed and roughly targets. The noise occurs multiplicative and additive. The main advantage of Wiener filter is to compute a statistical estimate of an unknown signal and a related signal that might consists of an unknown signal of interest that has been corrupted by noise. The estimate of unknown signal of interest is used to denoise the image.

The performance of average filter is satisfactory in these results in compare to median filter and Wiener filter. Gaussian filter is faster than median filter because multiplying and adding are probably faster than sorting. Wiener filter is good in removal of Poisson noise and Speckle noise but in Salt and Pepper noise, it is not so effective in compare to other filters.

\section{Result and Discussion}

The gray scale image of human brain is taken and noising and denoising is carried out using MATLAB.

For denoising linear filters like Gaussian filter $(3 \times 3)$ and average filter $(3 \times 3)$ and nonlinear filter like median filter $(3 \times 3)$ are used.

Calculation of PSNR and MSE values has been done using MATLAB functions $($ peaksnr $=$ psnr $(A, X) \&$ err $=$ immse $(\mathrm{A}, \mathrm{X})$, where $\mathrm{A}$ and $\mathrm{X}$ are noisy image and denoised image, respectively).

Table 3 shows the PSNR values. Higher the PSNR higher the filtering quality. The PSNR shows the performance of the filter and the RMSE describes the noisiness.

As shown in Table 3, the Gaussian filter has the highest PSNR for Gaussian noise. Similarly, the median filter removes Salt and Pepper noise at good rate. Wiener filter works good on Poisson and Speckle noise.

From Table 4, it is easy to understand the performance of filters. Gaussian filter has the lowest RMSE value for Gaussian noise. It demonstrates if the noise follows the Gaussian distribution or normal distribution Gaussian filter is significantly useful. 
Table 4. Results of RMSE

\begin{tabular}{lcccc}
\hline \multirow{2}{*}{ Noises } & \multicolumn{4}{c}{ Filters } \\
\cline { 2 - 5 } & Average & Median & Gaussian & Wiener \\
\hline Gaussian & 11.73 & 10.62 & 9.52 & 10.79 \\
$\begin{array}{l}\text { Salt \& } \\
\text { Pepper }\end{array}$ & 20.49 & 17.32 & 25.46 & 23.98 \\
Poisson & 9.65 & 7.48 & 6.11 & 5.19 \\
Speckle & 20.65 & 22.91 & 27.75 & 17.8 \\
\hline
\end{tabular}

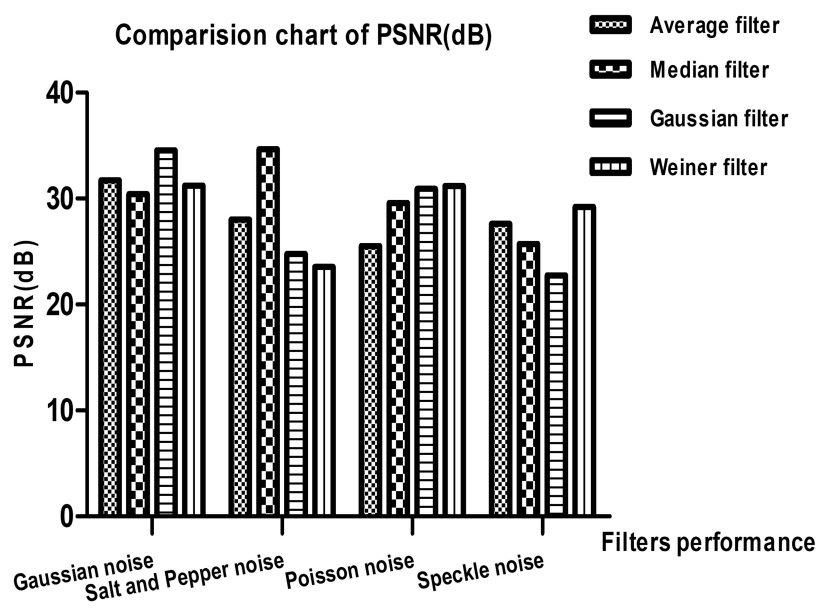

Figure 10. Comparison for PSNR.

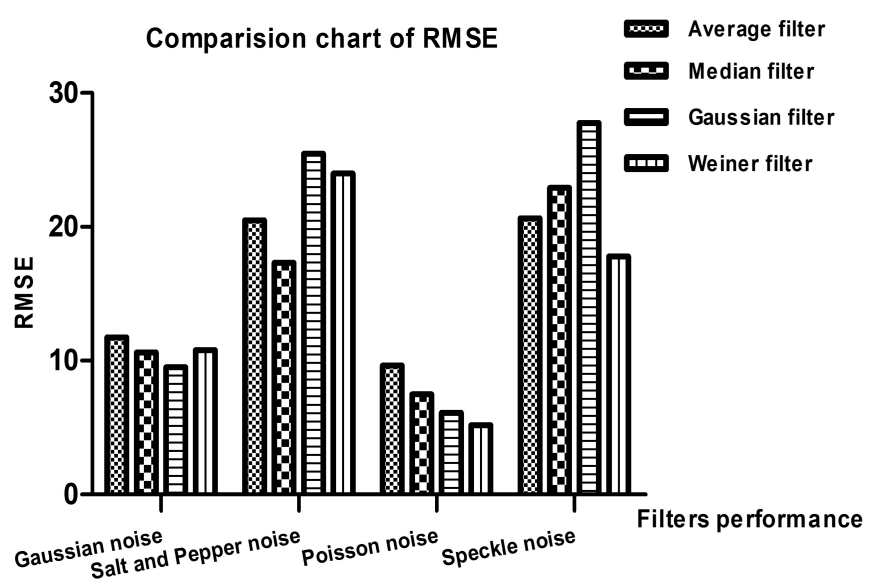

Figure 11. Comparison for RMSE.

The performance of median filter is better in Salt and Pepper noise. Wiener filter and Gaussian filters have almost similar effect on Poisson noise. Speckle noise has been more reduced by Winer filter followed by average filter. The comparison chart of PSNR, RMSE for four filters are shown in Figures 10 and 11 , respectively.
Based on performance and simulation results, we can say that Gaussian filter has good performance on Gaussian noise with median filter but lacking in case of Salt \& Pepper noise and Speckle and Poisson noise. Median filter and Wiener Filter have constantly good performance overall, but Wiener filter is less effective for Salt and Pepper noise than median filter. Average filter has good performance on Gaussian noise $(5 \times 5$ mask) and speckle noise. The performance of median filter is constantly good and Wiener filter is suitable to remove Poisson noise.

\section{Concluding Remarks}

Denoising medical images is generally quite a difficult. We here attempt to denoise four types of noises (Gaussian, Salt \& Pepper, Poisson, and Speckle noise) on human brain image using four filters (average, median, Gaussian, Wiener) in MATLAB environment. We have analyzed the performance of various filters for different noises. The PSNR shows the performance of the filter. Based on performance and simulation results, we could conclude that Gaussian filter and median filter were good on Gaussian and Salt \& Pepper noises, respectively.

The performance of Wiener filter was good on Poisson noise. This analysis can be further extended by including more noise types like anisotropic noise, Rayleigh noise, gamma noise, etc. and more denoising filters like adaptive filter, order static filter, geometric mean filter etc. One can also use hybrid filtering approach by using two or more filters. More study can be done in this field to provide more optimal solutions which results better and effective methodologies. Medical images like X-ray, MRI, ultrasound images can be used for denoising using various filtering algorithms.

\section{Conflict of Interest}

No potential conflict of interest relevant to this article was reported.

\section{Acknowledgements}

This research was supported by the Daegu University Research Grant.

\section{References}

[1] I. Singh and N. Neeru, "Performance comparison of various image denoising filters under spatial domain," Inter-

Performance Analysis of Denoising Algorithms for Human Brain Image | 
national Journal of Computer Applications, vol. 96, no. 19, pp. 21-30, 2014.

[2] P. Kumar Patidar, Lalit, B. Singh, and Gaurav Bagaria, "Image filtering using linear and non-linear filter for Gaussian noise," International Journal of Computer Applications, vol. 93, no. 8, pp. 29-34, 2014.

[3] V. Govindaraj and G. Sengottaiyan, "Survey of image denoising using different filters," International Journal of Science, Engineering and Technology Research, vol. 2, no. 2, pp. 344-351, 2013.

[4] H. S. Hwang and J. S. Oh, "Evolutionary design of morphology-based homomorphic filter for feature enhancement of medical images," International Journal of Fuzzy Logic and Intelligent Systems, vol. 9, no. 3, pp. 172177, 2009. https://doi.org/10.5391/IJFIS.2009.9.3.172

[5] L. Chen, and V. B. S. Prasath, "Measuring bone density connectivity using dual energy X-ray absorptiometry images," International Journal of Fuzzy Logic and Intelligent Systems, vol. 17, no. 4, pp. 235-244, 2017. http://dx.doi.org/10.5391/IJFIS.2017.17.4.235

[6] K. Park, H. S. Lee, and J. Lee, "Hybrid filter based on neural networks for removing quantum noise in low-dose medical X-ray CT images," International Journal of Fuzzy Logic and Intelligent Systems, vol. 15, no. 2, pp. 102-110, 2015. https://doi.org/10.5391/IJFIS.2015.15.2.102

[7] O. Bchir, M. M. B. Ismail, and H. Aljam, "Region-based image retrieval using relevance feature weights," International Journal of Fuzzy Logic and Intelligent Systems, vol. 18, no. 1, pp. 65-77, 2018. http://doi.org/10.5391/IJFIS. 2018.18.1.65

[8] MathWorks Inc., "Image Processing Toolbox," Available: http://www.mathworks.com/products/image.html

[9] K. A. Johnson and J. Alex Becker, "Atlas of the whole brain," Available: http://www.med.harvard.edu/aanlib/ home.html

[10] A. C. Bovick, Handbook of Image and Video Processing. San Diego, CA: Academic Press, 2000.

[11] S. Gupta and S. Gupta, "Despeckling of images using Wiener filter and hybrid median filter," International Journal of Computer Applications, vol. 154, no. 7, pp. 7-19, 2016.
[12] R. Verma and J. Ali, "A comparative study of various types of image noise and efficient noise removal techniques," International Journal of Advanced Research in Computer Science and Software Engineering, vol. 3, no. 10, pp. 617$622,2013$.

[13] Root-mean-square deviation, Available: https://en. wikipedia.org/wiki/Root-mean-square_deviation

[14] G. Kaur, R. Kumar, and K. Kainth, "A review paper on different noise types and digital image processing," International Journal of Advanced Research in Computer Science and Software Engineering, vol. 6, no. 6, pp. 562$565,2016$.

[15] P. S. Chahar and V. Thakare, "Performance comparison of various filters for removing Gaussian and Poisson noises," International Research Journal of Engineering and Technology, vol. 2, no. 5, pp. 1101-1105, 2015.

[16] A. K. Boyat and B. K. Joshi, "A review paper: noise models in digital image processing," Signal \& Image Processing: An International Journal, vol. 6, no. 2, pp. 63-75, 2015. https://doi.org/10.5121/sipij.2015.6206

[17] Q. Huynh-Thu and M. Ghanbari, "Scope of validity of PSNR in image/video quality assessment," Electronics Letters, vol. 44, no. 13, pp. 800-801, 2008. https://doi.org/ 10.1049/el:20080522

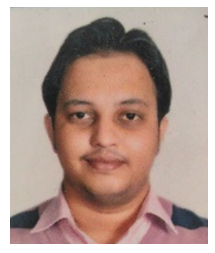

Nishant Chauhan received his Bachelor of Technology degree in computer science and engineering at Noida Institute of Engineering and Technology from AKTU University, India in 2012. He is a graduate student (master course) at Daegu University majoring Control Measurement. His research interests include intelligent control, fuzzy logic, image processing.

E-mail: nishantsep1090@daegu.ac.kr.

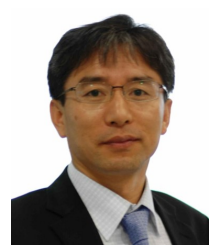

Byung-Jae Choi received his B.S. in Electronic Engineering, in 1987 from Kyungpook National University, Daegu. And he received his M.S. and Ph.D. degrees in Electrical and Electronic Engineering of KAIST, in 1989 and 1998, respectively. He is a Professor of School of Electronic and Electrical Engineering, Daegu University, Daegu, Korea, since 1999. His current research interests include intelligent control and its applications.

E-mail: bjchoi@daegu.ac.kr. 